\title{
OCORRÊNCIA DE PARASITÓIDES ASSOCIADOS À BROCA-DO-CAFÉ NO SUL DO ESPÍRITO SANTO-BRASIL
}

\author{
OCCURRENCE OF PARASITOID INSECTS ASSOCIATED TO THE COFFEE \\ BERRY BORER IN THE SOUTH OF THE STATE OF ESPÍRITO SANTO-BRAZIL
}

\author{
Leandro Pin Dalvi ${ }^{1}$; Dirceu Pratissoli ${ }^{1}$; Ricardo Antonio Polanczyk ${ }^{1}$; \\ Gilberto Santos Andrade ${ }^{2}$
}

\begin{abstract}
RESUMO
O objetivo deste trabalho foi verificar a ocorrência de insetos parasitóides, da broca-do-café, Hypothenemus hampei (Ferrari) (Coleoptera: Scolytidae) em plantios de café no Sul do Estado do Espírito Santo. Os parasitóides encontrados foram Prorops nasuta (Hym.: Bethilidae) e Cephalonomia stephanoderis (Hym.: Bethilidae), com níveis de parasitismo de até 33\%. A presença destes inimigos naturais sugere um potencial de regulação natural de $H$. hampei, e perspectivas para utilização em programas de Manejo Integrado de Pragas.
\end{abstract}

Palavras-chave: Controle biológico, Prorops nasuta, Cephalonomia stephanoderis, Hypothenemus hampei.

\begin{abstract}
The objective of this work was to verify the occurrence of parasitoids insects, of the coffee berry borer, Hypothenemus hampei (Ferrari) (Coleoptera: Scolytidae) in plantings of coffee in the South of Espirito Santo State. The found parasitoids were Prorops nasuta (Hym.: Bethilidae) and Cephalonomia stephanoderis (Hym.: Bethilidae), with levels of parasitism of up to 33\%. These natural enemies' presence suggests a potential of natural regulation of $\mathrm{H}$. hampei, and perspectives for its use in programs of Integrated Pest Management.

Key words: Biological control, Prorops nasuta, Cephalonomia stephanoderis, Hypothenemus hampei.
\end{abstract}

O Espírito Santo tem grande tradição na produção de café, e figura no cenário nacional brasileiro como o segundo maior produtor, sendo a cafeicultura a principal geradora de emprego e renda no setor agrícola do estado. Almejando mercados bem mais remunerados, porém muito exigentes, os produtores têm investido muito em melhorias na produtividade e principalmente na qualidade do produto.

A broca-do-café, Hypothenemus hampei (Ferrari) (Coleoptera: Scolytidae), inseto originário da África Central, é a praga mais importante da cultura em todo o mundo (Bustillo Pardey, 2005), causando a queda de frutos novos, perda de peso, e a maior parte dos defeitos na classificação quanto a tipo e bebida.

O método químico é amplamente utilizado, visando à redução de danos destes insetos, com aplicações de Endosulfan, um inseticida de largo espectro, o qual apresenta uma série de limitações, como o alto custo das aplicações, impacto no agroecossistema e o desenvolvimento de populações resistentes (Brun et al., 1989). Desse modo, são necessários estudos básicos visando desenvolver alternativas de controle, dentre as quais, destaca-se o controle biológico através da utilização de inimigos naturais (Santoro et al., 2005).

1 Laboratório de Entomologia, Departamento de Fitotecnia, Centro de Ciências Agrárias da Universidade Federal do Espírito Santo (Centro de Ciências Agrárias-Universidade Federal do Espírito Santo (CCA-UFES), Alto Universitário s/n, Alegre, ES. CEP 29500-000. E-mail: leandro-mpv@ @ca.ufes.br

2 Departamento de Entomologia-Universidad Federal de Viçosa, CEP: 36570-000, Viçosa - MG

* Autor para correspondência

Fecha de Recepción: 28 Junio 2007

Fecha de Aceptación: 05 Octubre 2007 
Quanto aos inimigos naturais da broca-do-café, os mais importantes são os parasitóides de origem africana, Prorops nasuta (Waterston) (Hymenoptera: Bethilidae), introduzido nas principais áreas produtoras do continente americano, além de Indonésia e Índia (Infante et al., 2003); Cephalonomia stephanoderis (Betrem) (Hymenoptera: Bethilidae), de grande eficiência na regulação desta praga (Lachaud et al., 2002); Heterospilus coffeicola (Schneiderknecht) (Hymenoptera: Braconidae), e, Phymastichus coffea (La salle) (Hymenoptera: Eulophidae), também introduzido no Brasil e demais áreas produtoras das Américas (Castillo et al., 2004); além do fungo entomopatogênico Beauveria bassiana, de ocorrência naturalmente em muitas regiões do país (Bustillo Pardey, 2005).

O presente trabalho teve como objetivo verificar a ocorrência de parasitóides associados à broca-do-café na região sul do Espírito Santo, visando a utilização destes agentes de controle biológico em futuros programas de Manejo Integrado de Pragas.

Durante o ano de 2006, foram realizadas coletas em 37 lavouras isoladas por barreiras naturais na

\section{REFERÊNCIAS BIBLIOGRÁFICAS}

BRUN, L., C. MARCILLAUD, V. GAUDICHON \& D SCUKLING. 1989. Endosulphan resistence in Hypothenemus hampei (Coleoptera: Scolytidae) in Caledonia. Journal of Economic Entomology 82: 1311-1316.

BUSTILLO PARDEY,A.E. 2005. El papel del control biológico en el manejo integrado de la broca del café, Hypothenemus hampei (Ferrari) (Coleoptera: Curculionidae: Scolytinae). Revista de la Academia Colombiana de Ciencias Exactas, Físicas y Naturales. 29: 55-68.

CASTILLO, A., F. INFANTE, G. LÓPEZ, \& J. TRUJILLO. 2004. Laboratory parasitism by Phymastichus coffea (Hymenoptera: Eulophidae) upon non-target bark beetles associated with coffee plantations. Florida Entomologist. 87: 274-277.
Região Sul do Espírito Santo. Em cada lavoura foram coletados 500 grãos brocados, em caminhamento zigue-zague com 50 pontos, estes grãos foram embalados em sacos de papel e trazidos ao setor entomologia do Núcleo de Desenvolvimento Científico e Tecnológico em Manejo Fitossanitário "NUDEMAFI" situado no campus do Centro de Ciências Agrárias da UFES (CCA-UFES), Alegre$\mathrm{ES}$, onde foram acondicionados em viveiros para emergência dos possíveis inimigos naturais.

Como resultado constatou-se a ocorrência dos parasitóides larvais: Prorops nasuta em material proveniente de cinco lavouras, com parasitismo variando de 2 a 33\%, e Cephalonomia stephanoderis, em uma lavoura com parasitismo de 3,6\%.

$\mathrm{O}$ fato de não serem relatadas liberações na região, indica que possivelmente os parasitóides a atingiram em sua dispersão natural, demonstrando que as condições ecológicas são favoráveis, permitindo seu estabelecimento neste agroecossistema. Estudos posteriores devem ser realizados para verificar o potencial destas duas espécies de parasitóides visando o controle da broca-do-café.

INFANTE, F., J. MUMFORD \& A. GARCÍA-BALLINAS. 2003. Predation by native arthropods on the African parasitoid Prorops nasuta (Hymenoptera: Bethylidae) in coffee plantations of Mexico. Florida Entomologist 86: 86-88.

LACHAUD, G.P., I.C.W. HARDY \& J.P. LACHAUD. 2002. Insect gladiators: competitive interactions between three species of bethylid wasps attacking the coffee berry borer, Hypothenemus hampei (Coleoptera: Scolytidae). Biological Control 25: 231-238.

SANTORO P.H., P.M.O.J. NEVES, R.Z. SILVA, S. AKIMI \& J. ZORZETTI. 2005. Produção de esporos de Beauveria bassiana (Bals). Vuill. num processo bifásico utilizando diferentes meios líquidos Semina: Ciências Agrárias 26: 313-320. 